Research Article

\title{
Predicting Stock Return with Economic Constraint: Can Interquartile Range Truncate the Outliers?
}

\author{
Zhifeng Dai $(\mathbb{D}$ and Xiaoming Chang \\ College of Mathematics and Statistics, Changsha University of Science and Technology, Changsha 410114, Hunan, China \\ Correspondence should be addressed to Zhifeng Dai; zhifengdai823@163.com
}

Received 20 March 2021; Accepted 12 June 2021; Published 21 June 2021

Academic Editor: José António Fonseca de Oliveira Correia

Copyright (c) 2021 Zhifeng Dai and Xiaoming Chang. This is an open access article distributed under the Creative Commons Attribution License, which permits unrestricted use, distribution, and reproduction in any medium, provided the original work is properly cited.

We find that imposing economic constraint on stock return forecasts based on the Interquartile Range of equity premium can significantly strengthen predictive performance. Specifically, we construct a judgment mechanism that truncates the outliers in forecasts of stock return. We prove that our constraint approach can realize more accurate predictive information relative to the unconstraint approach from the perspective of statistics and economics. In addition, the new constraint approach can effectively defeat CT constraint and CDA strategy. The three mixed models we proposed can further enhance the accuracy of prediction, especially the mixed model combined with our constraint approach. Finally, utilizing our new constraint approach can help investors obtain considerable economic gains. With the application of extension and robustness analysis, our results are robust.

\section{Introduction}

Stock is a major financial product in the financial market. The uncertainty of stock market fluctuation not only involves numerous economic behaviors but also contains many political factors. Therefore, researching the prediction of stock return has always been a considerable subject for many scholars and practitioners. However, predicting stock returns is notoriously difficult. The predictability of stock returns in sample has been confirmed by many studies (Campbell and Shiller [1]; Pontiff and Schall [2]; Dai et al. $[3,4]$; Ferson and Harvey [5]). In addition, according to Welch and Goyal [6], in terms of predicting out-of-sample stock returns, it is hard to find a certain model to go beyond the benchmark prediction model that treats stock returns as a constant. For this reason, a large number of scholars have proposed many predictors linked to macrofundamentals, which can enhance the predictability of out-of-sample stock returns, for example, oil-related variables (Liu et al. [7]), the variance risk premium (Bollerslev et al. [8,9]), technical indicators (Neely et al. [10]), economic policy uncertainty (Brogaard and Detzel [11]), manager sentiment (Jiang et al.
[12]), short interest (Rapach et al. [13]), news-implied volatility (Manela and Moreira [14]).

Numerous recent studies have verified that the singlepredictor model fails to beat the benchmark prediction model in the out-of-sample prediction of stock returns. The research of Pesaran and Timmermann [15] has shown that the uncertainty of parameters brings numerous issues to the prediction of stock returns, particularly, when the data contain a large number of noises. While more and more pieces of literature have found that economic constraints can improve the prediction of stock returns, there is no common view on the implementation of constraints. For example, to identify the parameter of the term structure model, Ang and Piazzesi [16] impose no arbitrage restrictions. Campbell and Thompson [17] require the negative predictive forecasts to become nonnegative and bind the sign of the slope coefficient in the predictive model of stock returns. Pástor and Stambaugh $[18,19]$ apply a priori information to ensure a negative correlation between expected returns and shocks of unexpected returns. Yi et al. [20] propose the cycledecomposing approach (CDA hereafter) that imposes restrictions on parameters. 
In this paper, we establish a new constraint approach to truncate stock return forecasts. We mainly report two types of constraints for comparison. Firstly, Campbell and Thompson [17] believe that when the stock return forecasts are negative, it should be truncated to be a nonnegative value. This is because it is unrealistic for a risk-averse investor to hold stocks when their expected returns are negative. Secondly, Yi et al. [20] propose the cycledecomposing approach to decompose economic variables into short-term bias and long-term trend. This approach achieves the limitation of model parameters.

The idea of this paper is designed to truncate prediction outliers generated by univariate predictive regression model. To realize this idea, we advance an approach that is related to the principle of boxplot. This approach needs to apply the concept of statistical quartile and uses the quartile difference (that is, the difference between the third quartile and the first quartile, also known as Interquartile Range) to deal with abnormal information. Therefore, we name this approach the Interquartile Range constraint (IQR, hereafter). The operation mechanism of the IQR constraint is setting up a reasonable interval for the stock return forecasts by utilizing the principle of boxplot when real stock return forecasts beyond the upper and lower range of this interval can be considered abnormal information.

Our main motivation for constructing this constraint approach is linked to but quite different from the CT constraint. CT constraint believes that investors will not hold stock when the expected returns are negative, but our approach finds that negative expected returns are still profitable. This is because investors are just unwilling to believe in extremely negative forecasts rather than being afraid of all negative forecasts. With this line, investors will not believe in extremely positive forecasts, too. Another motivation is that although it is unlikely to generate extreme return forecasts in the future, investors will pay special attention to the extreme changes of stock return prediction information. Therefore, they are more likely to react to these pieces of extreme information. In addition, there is always a positive relationship between return and risk; that is, extreme return forecasts are often accompanied by high risk. Consequently, investors will be more cautious about their portfolio allocation, and stock prices will respond to the behavior of investor. Collectively, the accuracy of stock return forecasts can be further improved after the IQR constraint model identifies return forecast outliers and rules out them. On the other hand, our motivation is partly inspired by Pettenuzzo et al. [21]. To make the portfolio performance more reasonable, they apply the Bayesian approach to restrict the Sharpe ratio to lie an upper and lower bound. The difference is that we use box graph instead of the Bayesian approach to determine the upper and lower bound.

For empirical analysis of our constraint approach, we apply 14 macroeconomic variables referred by Welch and Goyal [6] as the predictor of the single variable prediction regression model. Following Rapach and Zhou [22] and Dai and Zhu [23], we use the data from January 1927 to December 1947 as the initial period and employ January 1948 as the starting point for the recursive prediction of the expected return of the S\&P 500 index out of sample. We employ out-of-sample $R^{2}$ statistic to describe the forecasting performance of stock returns and examine the null hypothesis that $\mathrm{MSPE}^{b} \leq \mathrm{MSPE}^{i}$ with Clark and West [24] statistics. This test is often used to check whether $R_{\mathrm{OS}}^{2}$ is statistically significant.

The empirical results show that only the single-predictor model of SVAR and TBL can generate positive $R_{\mathrm{OS}}^{2}$ value, which is consistent with the previous literature results; that is, the predictive performance of macrofundamentals fails to exceed the historical average benchmark. In addition, compared with the original model, CT constraint and the CDA strategy can improve the prediction performance of 9 and 10 variables, respectively. Fortunately, we find that our constraint approach can generate larger $R_{\mathrm{os}}^{2}$ for all predictors relative to the original model. We also compute the average $R_{\mathrm{os}}^{2}$ value of each model as a comprehensive evaluation indicator for the corresponding predictive approach. The results reveal that the original model generates the average $R_{\mathrm{os}}^{2}$ value at $-0.502 \%$, the CT constraint increases it to $-0.272 \%$, the CDA strategy increases it to $0.094 \%$, and notably, the IQR constraint increases it to $0.261 \%$. Consequently, we conclude that the IQR constraint model can effectively improve the out-of-sample prediction ability compared with the original model, CT constraint, and the CDA strategy.

Furthermore, we further evaluate the forecasting performance of the model of interest by computing certainty equivalent return (CER) from an economics perspective. When the relative risk aversion coefficient is 3 , we calculate the difference for the certainty equivalent return (CER) obtained from the model of interest and the benchmark prediction model. Here, we find that the constraint model can lead to higher CER gains than the unconstraint model among most predictors. In particular, when investors use our constraint approach, the higher CER gains can be yielded compared to the unconstraint model among all predictors, which means that eliminating outliers in stock return forecasts can help investors to allocate their assets and create more substantial economic gains.

Following Dai and Zhu [25], we learned that the mixed model can also significantly strengthen the predictive performance. Therefore, based on the model of interest in this paper, we construct three mixed models, namely, CDA\&CT, CT\&IQR, and CDA\& IQR. The first model is to impose CT constraint on the prediction information of CDA strategy, the second model is to impose IQR constraint on the prediction information of CT constraint, and the last model is to impose IQR constraint on the prediction information of CDA strategy. The results show that the mixed model can yield more accurate prediction information and better economic gains than the unconstrained model and the competition model in this paper. Particularly, the mixed model connected with our constraint model shows the best prediction ability.

In addition, in terms of the business cycle, different risk aversion coefficients, transaction cost equal to 50, alternative weight ranges, and alternative out-of-sample periods, our results with the IQR constraint are all robust. In a word, our 
constraint model shows a stronger out-of-sample forecasting ability and creates considerable economic gains.

The arrangement of this paper is as follows: Section 2 describes the empirical data with summary statistics. Section 3 presents the constraint model and forecast strategy. Section 4 reports the empirical results for out-of-sample forecasting performance based on unconstraint model and constraint models. Section 5 shows some extensions and robustness test. Section 6 makes the conclusion.

\section{Empirical Data}

To predict out-of-sample stock returns, we employ 14 popular macroeconomic variables (we thank Amit Goyal for providing the latest data, which can be obtained from http://www.hec.unil.ch/agoyal/), which are mentioned in Welch and Goyal [6] who will update the data for the latest year. These variables are often used in many recent pieces of literature to predict stock returns [26-29]. We also use Amit Goyal's dataset to obtain the excess stock returns (we use the monthly return of S\&P 500 index minus the short T-bill rate). Next, we will give the description of 14 predictors.

(i) Log dividend-price ratio (DP): $\log$ of a 12-month moving sum of dividends paid on the S\&P 500 index minus the log of stock prices (S\&P 500 index)

(ii) Log dividend yield (DY): $\log$ of a 12-month moving sum of dividends minus the log of lagged stock prices

(iii) Log earnings-price ratio (EP): $\log$ of a 12-month moving sum of earnings on the S\&P 500 index minus the log of stock prices

(iv) Log dividend-payout ratio (DE): $\log$ of a 12-month moving sum of dividends minus the log of a 12 month moving sum of earnings

(v) Stock return variance (SVAR): sum of squared daily returns on the S\&P 500 index

(vi) Book-to-market ratio (BM): book-to-market value ratio for the Dow Jones Industrial Average

(vii) Net equity expansion (NTIS): ratio of a 12-month moving sum of net equity issues by NYSE-listed stocks to the total end-of-year market capitalization of NYSE stocks

(viii) Treasury bill rate (TBL): interest rate on a threemonth Treasury bill (secondary market)

(ix) Long-term yield (LTY): long-term government bond yield

(x) Long-term return (LTR): return on long-term government bonds

(xi) Term spread (TMS): long-term yield minus the Treasury bill rate

(xii) Default yield spread (DFY): difference between Moody's BAA- and AAA-rated corporate bond yields (xiii) Default return spread (DFR): long-term corporate bond return minus the long-term government bond return

(xiv) Inflation (INFL): calculated from the Consumer Price Index (CPI) for all urban consumers

This table reports summary statistics for excess stock return which was gained by the log return of S\&P 500 index minus the T-bill rate and for the 14 predictors obtained from the homepage of Amit Goyal: http://www.hec.unil.ch/ agoyal/. The sample period of this study ranges from 1927: 01 to $2018: 12$.

The summary statistics for 14 predictors and excess stock return from January 1927 through December 2018 are given in Table 1. We compute that the monthly average excess stock return can achieve $0.498 \%$, which is a considerable return. This is also an important reason why we are committed to studying the stock return forecasts. All data stem from Amit Goyal's dataset.

\section{Forecasting Regression Model and Constraint Models}

3.1. Forecasting Regression Model. According to the relevant literature, we utilize the following individual variable forecasting regression model to predict excess stock returns:

$$
\begin{aligned}
r_{t+1} & =\alpha_{i}+\beta_{i} x_{i t}+\varepsilon_{i . t+1}, \\
\varepsilon_{i, t+1} & \sim N\left(0, \sigma_{\varepsilon}^{2}\right),
\end{aligned}
$$

where $r_{t+1}$ represents the excess stock return at month $t+1$ over risk-free bill at month $t, x_{i, t}$ denotes the available variable for the $i$ th predictor at month $t$, and $\varepsilon_{i, t+1}$ is a disturbance term which is assumed to be independent and identically distributed, and the mean equals zero.

Based on the literature of Devpura et al. [30], we obtain out-of-sample excess stock return forecasts by applying a recursive estimation window. Particularly, the whole sample consisting of $T$ observations is divided into the first $M$ observations and T-M observations as the inner part of the sample and the outer part of the sample, respectively. Next, the following procedure is utilized to obtain out-of-sample forecast:

$$
\widehat{r}_{i, t+1}=\widehat{\alpha}_{i, t}+\widehat{\beta}_{i, t} x_{i, t} \text {, }
$$

where $\widehat{\alpha}_{i, t}$ and $\widehat{\beta}_{i, t}$ are the ordinary least squares (OLS) estimates of regression coefficients $\alpha$ and $\beta$ and each estimated result is generated by regressing $\left\{r_{t}\right\}_{t=2}^{m}$ on a constant and $\left\{x_{i, t}\right\}_{t=1}^{m-1}$. Continuing this step to make recursive prediction, we can obtain the out-of-sample forecast sequence, $\left\{\widehat{r}_{i, t}\right\}_{t=m+1}^{T}$, a total of T-M.

Following the paper of Goyal and Welch [31] and Hammerschmid and Lohre [32], after implementing the predictive regression model, we further apply the historical average benchmark model to evaluate the prediction accuracy of the original model and constraint model. The benchmark model actually assumes that $\beta_{i}$ in formula (1) is zero and is given as follows: 
TABLE 1: Summary statistics.

\begin{tabular}{|c|c|c|c|c|c|c|c|}
\hline Variable & Mean & Std. dev. & Median & Min & Max & Skewness & Kurtosis \\
\hline RETURN & 0.498 & 5.414 & 0.945 & -33.927 & 34.553 & -0.432 & 10.929 \\
\hline $\mathrm{DP}$ & -3.380 & 0.463 & -3.352 & -4.524 & -1.873 & -0.195 & 2.609 \\
\hline DY & -3.375 & 0.461 & -3.347 & -4.531 & -1.913 & -0.222 & 2.586 \\
\hline $\mathrm{EP}$ & -2.742 & 0.416 & -2.793 & -4.836 & -1.775 & -0.578 & 5.577 \\
\hline $\mathrm{DE}$ & -0.638 & 0.328 & -0.630 & -1.244 & 1.380 & 1.530 & 9.071 \\
\hline SVAR & 0.285 & 0.572 & 0.126 & 0.007 & 7.095 & 5.815 & 47.038 \\
\hline $\mathrm{BM}$ & 0.565 & 0.266 & 0.540 & 0.121 & 2.028 & 0.784 & 4.432 \\
\hline NTIS & 0.017 & 0.026 & 0.017 & -0.058 & 0.177 & 1.619 & 11.016 \\
\hline TBL & 3.389 & 3.086 & 2.930 & 0.010 & 16.300 & 1.094 & 4.330 \\
\hline LTY & 5.101 & 2.785 & 4.210 & 1.750 & 14.820 & 1.101 & 3.637 \\
\hline LTR & 0.472 & 2.446 & 0.310 & -11.240 & 15.230 & 0.578 & 7.567 \\
\hline TMS & 1.712 & 1.299 & 1.760 & -3.650 & 4.550 & -0.273 & 3.176 \\
\hline DFY & 1.123 & 0.690 & 0.900 & 0.320 & 5.640 & 2.499 & 11.998 \\
\hline DFR & 0.032 & 1.368 & 0.050 & -9.750 & 7.370 & -0.373 & 10.561 \\
\hline INFL & 0.242 & 0.531 & 0.241 & -2.055 & 5.882 & 1.083 & 16.929 \\
\hline
\end{tabular}

$$
\mathrm{HA}(t)=\bar{r}_{t}=\frac{1}{t} \sum_{i=1}^{t} r_{i} .
$$

3.2. Constraint Models. To compare our constraint model, we also consider two prevailing constraint models as competition model. The first one is proposed by Campbell and Thompson [17] who believe that it is difficult for investors to hold stocks when the forecast signal is negative (that is, the stock excess return forecast is negative), thus constraining the negative return forecasts to zero. The specific model is given as follows:

$$
\widehat{r}_{i, t+1}^{\mathrm{CT}}= \begin{cases}0, & \text { if } \widehat{r}_{i, t+1}<0, \\ \widehat{r}_{i, t+1}, & \text { else. }\end{cases}
$$

The second one is put forward by Yi et al. [20]. They decompose the predictor into the long-term mean part and the short-term deviation part and then carry out the least square regression for the decomposed content. Finally, compute stock return forecast based on the corresponding return forecast of the two parts by utilizing the simple average combination strategy. The specific operation process is given as follows:

$$
\left\{\begin{array}{c}
x_{i, t \text { mean }}^{C}=\frac{1}{C} \sum_{\tau=t-C+1}^{t} x_{i, \tau}, \\
x_{i, t, \mathrm{dev}}^{C}=x_{i, t}-x_{i, t \text { mean }}^{C},
\end{array}\right.
$$

where $C$ is the lag order of the long-cycle term and equals 12 . $x_{i, \tau}$ denotes the available variable for the $i$ th predictor. $x_{i, t \text {,mean }}^{C}$ denotes the long-term mean of the $i$ th at month $t$. $x_{i, t, \mathrm{dev}}^{C}$ denotes the short-term deviation of the $i$ th at month $t$.

$$
\left\{\begin{array}{l}
\widehat{r}_{i, t+1, \text { mean }}^{C}=\widehat{\alpha}_{i, t, \text { mean }}+\widehat{\beta}_{i, t, \text { mean }} x_{i, t, \text { mean }}^{C}, \\
\widehat{r}_{i, t+1, \text { dev }}^{C}=\widehat{\alpha}_{i, t, \text { dev }}+\widehat{\beta}_{i, t, \mathrm{dev}} x_{i, t, \mathrm{dev}}^{C}, \\
\widehat{r}_{i, t+1}^{\mathrm{CDA}}=0.5\left(\widehat{r}_{i, t+1, \text { mean }}^{C}+\widehat{r}_{i, t+1, \mathrm{dev}}^{C}\right)
\end{array}\right.
$$

where $\widehat{\alpha}_{i, t \text {,mean }}, \widehat{\beta}_{i, t \text {,mean }}, \widehat{\alpha}_{i, t, \mathrm{dev}}$, and $\widehat{\beta}_{i, t, \mathrm{dev}}$ are the ordinary least squares (OLS) estimates of regression coefficients $\alpha$ and $\beta$ and generated by regressing $\left\{r_{t}\right\}_{t=2}^{m}$ on a constant and $\left\{x_{i, t}\right\}_{t=1}^{m-1} \cdot \widehat{r}_{i, t+1}^{\mathrm{CDA}}$ is the ultimately out-of-sample predictive content we need.

We advance a new constraint model which applies the concept of statistical quartile and uses the quartile difference (that is, the difference between the third quartile and the first quartile, also known as Interquartile Range) to deal with abnormal forecasts. Therefore, we name this approach the Interquartile Range constraint (IQR, hereafter). More specifically, we establish a judgment mechanism for the new constraint model, which sets an interval for the original prediction value without outliers. We give its mathematical form as follows:

$$
\widehat{r}_{i, t+1}^{\mathrm{IQR}}= \begin{cases}r_{i, t}-3 * \mathrm{IQR}_{t}, & \text { if } \widehat{r}_{i, t+1}<r_{i, t}-3 * \mathrm{IQR}_{t} \\ r_{i, t}+3 * \mathrm{IQR}_{t}, & \text { if } \widehat{r}_{i, t+1}>r_{i, t}-3 * \mathrm{IQR}_{t} \\ \widehat{r}_{i, t+1}, & \text { otherwise, }\end{cases}
$$

where $\widehat{r}_{i, t+1}^{\mathrm{IQR}}$ is the excess stock return forecast of our new constraint model for month $t+1$ and $i$ th predictor. $\mathrm{IQR}_{t}$ denotes the Interquartile Range of actual stock return at month $t$. $\widehat{r}_{i, t+1}$ represents the excess stock return forecast at month $t+1$ based on regression in (2). Notably, we choose three times the Interquartile Range as the criterion for truncating outliers to acquire economic constraint more 
reasonable and restrictive (in fact, we do not consider using other multiples of the Interquartile Range, because twice the Interquartile Range may truncate a large number of normal forecasts. Conversely, four times the Interquartile Range expands the criterion of truncation and loses its restrictiveness).

\section{Empirical Results for Equity Premium Forecasts}

In this part, we evaluate the forecasting performance of the predictive model from an economic and statistic point of view. The out-of-sample period is $1948: 01-2018: 12$.

\subsection{Assessing the Out-of-Sample Statistics Performance.} We employ a widely accepted approach to evaluate the outof-sample forecasting performance of constraint and unconstraint models. This approach generally compares the forecasting models with the historical average benchmark model to weigh the accuracy of return forecasts (see, e.g., [32-37]). The method is $R^{2}\left(R_{\mathrm{OS}}^{2}\right)$ statistic which was proposed by Campbell and Thompson [17].

$R^{2}$ denotes as the percentage reduction for the mean squared prediction error of the forecasting model relative to the mean square prediction error of the benchmark model. Next, we calculate the out-of-sample $R^{2}\left(R_{\text {OS }}^{2}\right)$ statistic.

$$
R_{\mathrm{os}}^{2}=1-\frac{\operatorname{MSPE}^{i}}{\operatorname{MSPE}^{b}}=1-\frac{(1 / k) \sum_{t=M+1}^{M+k}\left(r_{t}-\widehat{r}_{t}^{i}\right)^{2}}{(1 / k) \sum_{t=M+1}^{M+k}\left(r_{t}-\bar{r}_{t}\right)^{2}} .
$$

As can be seen from the above formula, $R_{\mathrm{OS}}^{2}$ is a positive value, which means that the model of interest will produce more accurate forecasting information than the benchmark model. Following previous literature of Buncic and Tischhauser [38], we further apply the Clark and West [24] test (CW hereafter) to examine whether the forecasting regression model has a lower MSPE relative to the benchmark model. The null hypothesis is that $\mathrm{MSPE}^{b} \leq \mathrm{MSPE}^{i}$ and the alternative hypothesis is that $\mathrm{MSPE}^{b}>\mathrm{MSPE}^{i}$. The CW statistic is a modification of Diebold and Mariano [39] statistic, which is convenient to evaluate statistical significance. To calculate the CW statistic, we first define the MSPE-adjusted statistic:

$$
f_{t}=\left(r_{t}-\bar{r}_{t}\right)^{2}-\left(r_{t}-\widehat{r}_{t}\right)^{2}+\left(\bar{r}_{t}-\widehat{r}_{t}\right)^{2}
$$

Next, we regress $\left\{f_{i, t}\right\}_{t=m}^{T}$ to a constant so that we can get the $t$-statistics of the constant. Then, the standard normal distribution is used to calculate the $p$ value of one side (upper tail) test. The $p$ value represents the statistical significance that the model of interest has a smaller mean square prediction error compared with the benchmark model.

This table shows the out-of-sample statistical performance of the original (no constraint) model and constraint models based on 14 macroeconomic predictors. We evaluate the accuracy of out-of-sample stock return forecast by employing $R_{\mathrm{OS}}^{2} \cdot R_{\mathrm{OS}}^{2}$ computes the reduction in MSPE (mean squared prediction error) for the predictive regression model relative to the benchmark model. The statistical significance of $R_{\mathrm{OS}}^{2}$ is examined by employing Clark and West [24] test. $* * *, * *$, and $*$ mean significance at the $1 \%, 5 \%$, and $10 \%$ levels, respectively.

Table 2 reports the out-of-sample statistical performance with regard to the original model and constraint models. We apply $R_{\mathrm{OS}}^{2}$ and CW statistics to evaluate statistical performance and statistical significance, respectively. Our empirical results reveal that only SVAR, TBL, and TMS can yield positive $R_{\mathrm{OS}}^{2}$ based on univariate predictive regression, which once again validates the research of Welch and Goyal [6] who believed that it is difficult for individual predictor to obtain better predictive performance relative to the historical average.

Comparing the CT constraint and the CDA strategy to the original model, the results display that there are 10 and 12 variables that produce larger $R_{\mathrm{OS}}^{2}$, respectively. On the other hand, 6 and 7 out of them are positive, respectively. At the same time, we find that the predictive performance of all individual predictors can effectively strengthen after implementing IQR constraint to deal with the outliers in the original model. What is more, our new constrain model can yield 11 positive $R_{\mathrm{OS}}^{2}$ relative to the benchmark model. When we apply CW test, DP, DY, EP, NTIS, TBL, LTY, and TMS significantly outperform the historical average model. More specifically, based on the whole sample, the new constraint model we submitted can promote $R_{\mathrm{OS}}^{2}$ for DP from $-0.074 \%$ to $0.653 \%$, promote it for DY from $-0.389 \%$ to $0.252 \%$, promote it for SVAR from $0.154 \%$ to $0.836 \%$, promote it for NTIS from $-0.633 \%$ to $0.313 \%$, promote it for TBL from $0.067 \%$ to $0.844 \%$, promote it for TMS from $0.103 \%$ to $0.994 \%$, and promote it for INFL from $-0.060 \%$ to $0.727 \%$. We also calculate the average $R_{\mathrm{OS}}^{2}$ to evaluate the comprehensive predictive ability of the corresponding predictive model and find that the CT constraint and CDA strategy can promote the average $R_{\mathrm{OS}}^{2}$ from $-0.502 \%$ to $-0.272 \%$ and $0.094 \%$, respectively, while our IQR constraint model generates the largest average of $R_{\mathrm{OS}}^{2}$ at $0.261 \%$. Consequently, we believe that the IQR constraint model can effectively improve the out-of-sample predictive accuracy compared with the original model, CT constraint, and CDA strategy.

\subsection{Assessing the Out-of-Sample Economic Performance.} We have testified that the constraint approach significantly outperforms the benchmark model in the prediction of stock returns from the point of view of statistics. To describe stock return forecasts with utility-related indicator from an economic perspective (Campbell and Thompson [17]; Dai and Kang [40]), we further evaluate the forecasting performance of stock return by calculating the certainty equivalent return (CER). We assume that a mean-variance investor allocates their assets among the S\&P 500 index and the risk-free property. To maximize the CER, we set the optimal weight of the stock in the portfolio during $t+1$ as follows:

$$
\omega_{i, t}^{*}=\frac{1}{\gamma} \frac{\widehat{r}_{i, t+1}}{\widehat{\sigma}_{t+1}^{2}}
$$


TABLE 2: Forecasting performance of existing models evaluated by $R_{\mathrm{OS}}^{2}$.

\begin{tabular}{lcccc}
\hline & Original & CT & CDA & IQR \\
\hline DP & $-0.074^{*}$ & $\mathbf{0 . 0 9 4}^{*}$ & $\mathbf{0 . 3 5 1}^{*}$ & $\mathbf{0 . 6 5 3}^{* *}$ \\
DY & $-0.389^{* *}$ & $\mathbf{0 . 0 8 8}^{* *}$ & $\mathbf{0 . 3 0 1}^{* *}$ & $\mathbf{0 . 2 5 2}^{* *}$ \\
EP & $-1.463^{*}$ & $-\mathbf{0 . 5 8 0 ^ { * * }}$ & $\mathbf{0 . 1 5 0 ^ { * }}$ & $-\mathbf{0 . 7 9 3} 3^{* *}$ \\
DE & -1.458 & $-\mathbf{1 . 2 1 0}$ & $-\mathbf{0 . 6 8 6}$ & $-\mathbf{0 . 6 9 4}$ \\
SVAR & 0.154 & 0.076 & 0.123 & $\mathbf{0 . 8 3 6}$ \\
BM & -1.467 & $-\mathbf{1 . 0 0 0}$ & $-\mathbf{0 . 0 6 8}$ & $-\mathbf{0 . 8 0 0}$ \\
NTIS & -0.633 & $-\mathbf{0 . 6 3 2}$ & $-\mathbf{0 . 4 9 2}$ & $\mathbf{0 . 3 1 3 ^ { * }}$ \\
TBL & $0.067^{*}$ & $\mathbf{0 . 2 4 9}$ & $\mathbf{0 . 2 7 7 ^ { * }}$ & $\mathbf{0 . 8 4 4 ^ { * * }}$ \\
LTY & $-0.677^{*}$ & $\mathbf{0 . 2 6 7 ^ { * * }}$ & $\mathbf{1 . 6 8 9 * * *}$ & $\mathbf{0 . 0 6 9 ^ { * * }}$ \\
LTR & -0.761 & $-\mathbf{0 . 6 4 1}$ & $\mathbf{0 . 6 4 6 * *}$ & $\mathbf{0 . 0 8 3}$ \\
TMS & 0.103 & 0.098 & -0.241 & $\mathbf{0 . 9 9 4} 4^{* *}$ \\
DFY & -0.169 & -0.169 & $-\mathbf{0 . 1 1 8}$ & $\mathbf{0 . 6 3 4}$ \\
DFR & -0.205 & -0.409 & -0.305 & $\mathbf{0 . 5 3 0}$ \\
INFL & -0.060 & $-\mathbf{0 . 0 4 0}$ & -0.309 & $\mathbf{0 . 7 2 7}$ \\
Average & -0.502 & $-\mathbf{0 . 2 7 2}$ & $\mathbf{0 . 0 9 4}$ & $\mathbf{0 . 2 6 1}$ \\
\hline
\end{tabular}

where $\gamma$ represents the risk aversion coefficient of investor, $\widehat{r}_{i, t+1}$ is the stock return forecast based on a different predictive model, and $\widehat{\sigma}_{t+1}^{2}$ is the variance prediction of stock return time series during $t+1$. For risk aversion coefficient, $\gamma=3$, as in Rapach et al. [26]. We followed Campbell and Thompson [17] with a five-year rolling window to compute variance forecasts. Meanwhile, we assign $1-\omega_{i, t+1}^{*}$ to riskfree bills. In addition, following the information in the relevant literature (e.g., Huang et al. [34]; Pan et al. [23]), in order to prevent more than $50 \%$ of short selling and financial leverage, $\omega_{i, t}^{*}$ is limited to -0.5 to 1.5 . The portfolio returns of investor in stocks and risk-free bills are defined as follows:

$$
R_{i, t+1}=\omega_{i, t}^{*} r_{t+1}+R_{f, t+1} \text {, }
$$

where $R_{f, t+1}$ represents risk-free rate. Finally, we employ the following formula to solve CER:

$$
\mathrm{CER}_{i}=\widehat{\mu}_{i}-0.5 \gamma \widehat{\sigma}_{i}^{2}
$$

where $\widehat{\mu}_{i}$ and $\widehat{\sigma}_{i}^{2}$ represent the mean and variance of the portfolio of stocks and risk-free bills, respectively. CER gains are the metric of economic performance that equals portfolio management fees that mean-variance investors are willing to pay to obtain predictive formation relative to the historical average benchmark. Accordingly, the result of CER gains is the difference between the CER of the model of interest and the CER of the benchmark model.

This table shows the out-of-sample portfolio performance of the original (no constraint) model and constraint models based on 14 macroeconomic predictors. We employ the certainty equivalent return (CER) gains to remark the performance of the portfolio. CER is a utility-related indicator which is derived from investors investing their assets between stock and T-bill rate. The CER gains indicate the difference between the forecasting model we applied and the benchmark forecast model. We use a risk aversion coefficient equal to 3 and limit the weight to -0.5 to 1.5 to calculate the CER. Furthermore, the CER gains should be multiplied by 1200 as an annualized percentage value.
Table 3 reports the economic performance of the stock return forecasts assessed in terms of CER gains of an investor who applies the given constraint model and original model relative to the benchmark forecasting model. In this table, the CER gains are multiplied by 1200 to represent the annualized percent values. Comparing the original model with constraint models based on economic performance, the result reveals that the CT constraint model fails to significantly strengthen CER gains. In addition, the CDA strategy can effectively promote CER gains. Delightfully, the CER gains are further promoted compared to the original model and CT constraint model when investors use our constraint model. It is noteworthy that all predictors improve their economic performance after implementing the IQR constraint model. What is more, 10 of 14 the CER gains are greater than zero, which means that our constraint model can strongly beat the benchmark model in terms of economic performance. More specially, by the application of IQR constraint model, the CER gains promote it from $-0.207 \%$ to $0.216 \%$ for SVAR, from $0.530 \%$ to $0.919 \%$ for NTIS, from $1.497 \%$ to $1.755 \%$ for TBL, from $0.622 \%$ to $0.864 \%$ for LTY, from $0.591 \%$ to $0878 \%$ for LTR, and from $1.547 \%$ to $1.967 \%$ for TMS. In a word, implementing the IQR constraint approach can help investors gain more economic gains.

4.3. Mixing Existing Model. There are many studies that have shown that combination forecasting can effectively promote the prediction accuracy of stock returns. The combination approach includes two modes: one is to combine the prediction variables (see, e.g., Kelly and Pruitt [41]; Bahrami et al. [42]; Pettenuzzo et al. [21]), and the other is to combine the popular models (see, e.g., [43-48]). Following the literature of Dai and Zhu [25], we employ a mixed model by mixing existing forecasting models to further study stock return forecasts. In this paper, existing forecasting models refer to the CT constraint model, CDA strategy, and IQR constraint model, respectively. With regard to the first mixed model, we consider the reorganization of CT constraints and CDA strategy, namely, the CDA\&CT constraint model. The specific implementation steps are as follows:

$$
\widehat{r}_{i, t+1}^{\mathrm{CDA} \& \mathrm{CT}}=\max \left(0, \widehat{r}_{i, t+1}^{\mathrm{CDA}}\right) \text {. }
$$

The second mixed model is to impose the IQR constraint on the stock return forecasts of the CT constraint, namely, the CT\&IQR constraint model. The details are as follows:

$$
\widehat{r}_{i, t+1}^{\mathrm{CT} \& \mathrm{IQR}}= \begin{cases}r_{i, t}-3 * \mathrm{IQR}_{t}, & \text { if } \widehat{r}_{i, t+1}^{\mathrm{CT}}<r_{i, t}-3 * \mathrm{IQR}_{t}, \\ r_{i, t}+3 * \mathrm{IQR}_{t}, & \text { if } \widehat{r}_{i, t+1}^{\mathrm{CT}}>r_{i, t}+3 * \mathrm{IQR}_{t}, \\ \widehat{r}_{i, t+1}^{\mathrm{CT}}, & \text { otherwise. }\end{cases}
$$

For the last mixed forecasting model, we consider applying the IQR constraint to eliminate outliers obtained by the CDA strategy, namely, the CDA\&IQR constraint model. 
TABLE 3: Portfolio performance of existing models evaluated by CER gains.

\begin{tabular}{lcccc}
\hline & Original & CT & CDA & IQR \\
\hline DP & -1.711 & $-\mathbf{1 . 1 2 7}$ & $\mathbf{0 . 4 0 6}$ & $-\mathbf{1 . 5 4 1}$ \\
DY & -1.973 & $-\mathbf{1 . 1 4 1}$ & $-\mathbf{0 . 2 0 9}$ & $-\mathbf{1 . 9 6 3}$ \\
EP & 0.417 & $\mathbf{0 . 5 3 7}$ & 0.193 & $\mathbf{0 . 5 4 5}$ \\
DE & -0.270 & $-\mathbf{0 . 2 0 7}$ & $\mathbf{0 . 2 2 0}$ & $\mathbf{0 . 0 4 2}$ \\
SVAR & -0.202 & -0.202 & $\mathbf{0 . 1 9 4}$ & $\mathbf{0 . 2 1 6}$ \\
BM & -2.182 & $-\mathbf{1 . 4 2 8}$ & $-\mathbf{0 . 6 3 8}$ & $-\mathbf{2 . 1 3 6}$ \\
NTIS & 0.530 & 0.530 & 0.454 & $\mathbf{0 . 9 1 9}$ \\
TBL & 1.497 & 1.479 & $\mathbf{1 . 8 8 2}$ & $\mathbf{1 . 7 5 5}$ \\
LTY & 0.622 & $\mathbf{0 . 9 5 9}$ & $\mathbf{3 . 2 2 0}$ & $\mathbf{0 . 8 6 4}$ \\
LTR & 0.591 & 0.591 & $\mathbf{1 . 3 9 4}$ & $\mathbf{0 . 8 7 8}$ \\
TMS & 1.547 & $\mathbf{1 . 5 5 5}$ & 0.493 & $\mathbf{1 . 9 6 7}$ \\
DFY & -0.389 & $-\mathbf{0 . 3 6 1}$ & $\mathbf{0 . 0 1 9}$ & $-\mathbf{0 . 0 8 2}$ \\
DFR & 0.634 & 0.569 & 0.076 & $\mathbf{0 . 8 7 4}$ \\
INFL & 0.233 & $\mathbf{0 . 2 7 2}$ & 0.165 & $\mathbf{0 . 5 2 3}$ \\
\hline
\end{tabular}

$$
\widehat{r}_{i, t+1}^{\mathrm{CDA} \& \mathrm{IQR}}= \begin{cases}r_{i, t}-3 * \mathrm{IQR}_{t}, & \text { if } \widehat{r}_{i, t+1}^{\mathrm{CDA}}<r_{i, t}-3 * \mathrm{IQR}_{t}, \\ r_{i, t}+3 * \mathrm{IQR}_{t}, & \text { if } \widehat{r}_{i, t+1}^{\mathrm{CDA}}>r_{i, t}+3 * \mathrm{IQR}_{t} \\ \widehat{r}_{i, t+1}^{\mathrm{CDA}}, & \text { otherwise. }\end{cases}
$$

This table shows the out-of-sample statistical performance of the mixed existing constraint models based on 14 macroeconomic predictors. We evaluate the accuracy of outof-sample stock return forecast by employing $R_{\mathrm{OS}}^{2} \cdot R_{\mathrm{OS}}^{2}$ computes the reduction in MSPE (mean squared prediction error) for the predictive regression model relative to the benchmark model. The statistical significance of $R_{\mathrm{OS}}^{2}$ is examined by employing Clark and West [24] test. $* * *$, $* *$, and $*$ mean significance at the $1 \%, 5 \%$, and $10 \%$ levels, respectively.

Table 4 presents the statistical performance of the mixed models based on the existing model; similarly, $R_{\mathrm{OS}}^{2}$ is used for evaluation. The results reveal that utilizing the CDA\&CT model can enhance 10 of $14 R_{\mathrm{OS}}^{2}$ compared to the original model. After implementing the CT\&IQR model, we find that all of the 14 macroeconomic predictors are able to significantly intensify concerning out-of-sample prediction ability. What is more, the CT\&IQR constraint model can yield 12 positive $R_{\mathrm{OS}}^{2}$ relative to benchmark model. When we apply the CDA\&IQR constraint model, all of $R_{\mathrm{OS}}^{2}$ are positive, which means that the CDA\&IQR constraint model can significantly outperform the historical average model. Notably, the prediction performance of some predictors can be greatly improved by utilizing CDA\&IQR constraint, such that $R_{\text {OS }}^{2}$ promotes it from $-1.463 \%$ to $0.839 \%$ for $\mathrm{EP}$, from $-0.389 \%$ to $1.004 \%$ for DY, from $-0.074 \%$ to $1.011 \%$ for DP, from $0.067 \%$ to $1.066 \%$ for TBL, from $-0.761 \%$ to $1.411 \%$ for LTR, and most notably, from $-0.677 \%$ to $2.384 \%$ for LTY. On the other hand, the average $R_{\text {OS }}^{2}$ for the three mixed models arrives at $0.140,0.505$, and 0.850 , respectively, which is significantly higher than the original model. In addition, we find that the average $R_{\mathrm{OS}}^{2}$ of each mixed model is effectively higher than the corresponding existing model.
TABLE 4: Forecasting performance of mixed existing models evaluated by $R_{\mathrm{OS}}^{2}$.

\begin{tabular}{|c|c|c|c|}
\hline & CDA\&CT & CT\&IQR & CDA\&IQR \\
\hline DP & $0.439^{* *}$ & $0.821^{* *}$ & $1.011^{* *}$ \\
\hline DY & $0.329^{* *}$ & $0.779^{* *}$ & $1.004^{* *}$ \\
\hline EP & $0.428^{* *}$ & $0.126^{* *}$ & $0.839^{* *}$ \\
\hline $\mathrm{DE}$ & -0.377 & -0.446 & 0.012 \\
\hline SVAR & 0.073 & 0.857 & $0.892^{*}$ \\
\hline $\mathrm{BM}$ & -0.042 & -0.319 & $0.614^{*}$ \\
\hline NTIS & -0.488 & $0.314^{*}$ & 0.433 \\
\hline TBL & $0.242^{*}$ & $1.027^{* *}$ & $1.066^{* *}$ \\
\hline LTY & $1.461^{* * *}$ & $1.013^{* *}$ & $2.384^{* * *}$ \\
\hline LTR & $0.767^{* * *}$ & 0.203 & $1.411^{* * *}$ \\
\hline TMS & -0.153 & $0.990^{* *}$ & 0.581 \\
\hline DFY & -0.118 & 0.634 & 0.680 \\
\hline DFR & -0.295 & 0.327 & 0.487 \\
\hline INFL & -0.309 & 0.747 & 0.482 \\
\hline Average & 0.140 & 0.505 & 0.850 \\
\hline
\end{tabular}

The above evidence shows that the mixed model owns more accurate prediction contents than the existing forecasting model in this paper.

This table shows the out-of-sample portfolio performance of the original (no constraint) model and constraint models based on 14 macroeconomic predictors. We employ the certainty equivalent return (CER) gains to remark the performance of the portfolio. CER is a utility-related indicator which is derived from investors investing their assets between stock and T-bill rate. The CER gains indicate the difference between the forecasting model we applied and the benchmark forecast model. We use risk aversion coefficient equal to 3 and limit the weight to -0.5 to 1.5 to calculate the CER. Furthermore, the CER gains should be multiplied by 1200 as an annualized percentage value.

Table 5 reports the economic performance of the mixed models, assessed in terms of CER gains. The results of the mixed model show that most of the CER gains are greater than zero, which implies that the combination of existing models can effectively exceed the benchmark forecasting model. We further compare the CER gains of the mixed model with the original model and the existing models and find that the mixed model is obviously superior to not only the original model but also the corresponding existing model. In terms of the three mixed models in this paper, CDA\&IQR model has the best portfolio performance. Note that CDA\&IQR model has generated 12 positive CER gains and achieved more than 1\% CER gains with regard to LTR, TBL, and LTY. In particular, the CDA\&IQR model promotes the CER gains for LTR from $0.591 \%$ to $1.675 \%$, promotes it for TBL from $1.497 \%$ to $2.159 \%$, and promotes it for LTY from $0.622 \%$ to $3.379 \%$. Overall, the mixed model has a strong predictive utility in terms of portfolios.

\section{Extensions and Robustness Analysis}

In this section, in order to further evaluate the results of Section 4, we propose several extensions and robustness tests which include the out-of-sample performance over the business cycle, CER for alternative risk aversion coefficient, 
TABLE 5: Portfolio performance of mixed models evaluated by CER gains.

\begin{tabular}{lccc}
\hline & CDA\&CT & CT\&IQR & CDA\&IQR \\
\hline DP & $\mathbf{0 . 4 8 0}$ & $-\mathbf{0 . 9 5 6}$ & $\mathbf{0 . 4 7 0}$ \\
DY & $-\mathbf{0 . 1 9 0}$ & $-\mathbf{1 . 0 7 4}$ & $-\mathbf{0 . 0 8 0}$ \\
EP & 0.411 & $\mathbf{0 . 6 6 5}$ & 0.316 \\
DE & $\mathbf{0 . 2 3 4}$ & $\mathbf{0 . 1 0 6}$ & $\mathbf{0 . 4 0 9}$ \\
SVAR & $\mathbf{0 . 1 9 4}$ & $\mathbf{0 . 2 1 6}$ & $\mathbf{0 . 4 7 2}$ \\
BM & $-\mathbf{0 . 6 3 8}$ & $-\mathbf{1 . 3 7 1}$ & $-\mathbf{0 . 5 3 4}$ \\
NTIS & 0.454 & $\mathbf{0 . 9 1 9}$ & $\mathbf{0 . 8 7 4}$ \\
TBL & $\mathbf{1 . 8 4 6}$ & $\mathbf{1 . 7 3 7}$ & $\mathbf{2 . 1 5 9}$ \\
LTY & $\mathbf{2 . 9 3 2}$ & $\mathbf{1 . 2 0 1}$ & $\mathbf{3 . 3 7 9}$ \\
LTR & $\mathbf{1 . 3 7 6}$ & $\mathbf{0 . 8 7 8}$ & $\mathbf{1 . 6 7 5}$ \\
TMS & 0.526 & $\mathbf{1 . 9 7 5}$ & 0.870 \\
DFY & $\mathbf{0 . 0 1 9}$ & $-\mathbf{0 . 0 5 3}$ & $\mathbf{0 . 3 2 7}$ \\
DFR & 0.076 & $\mathbf{0 . 8 0 8}$ & 0.347 \\
INFL & 0.165 & $\mathbf{0 . 5 6 2}$ & $\mathbf{0 . 4 7 0}$ \\
\hline
\end{tabular}

CER with transaction cost, combination forecast approaches, and the predictive power of the model of interest based on different out-of-sample periods.

5.1. $R_{\text {OS }}^{2}$ over Business Cycle. Why can the IQR constraint model predict stock returns? Cochrane [49] and Fama and French [50] have shown that the predictability of the stock market is affected by the business cycle. One explanation is that investors take on high risk during the recession, which makes investors desire for higher risk premium, thus creating the predictability of stock returns. On the other hand, compared with the expansion period, the recession period can obtain better prediction performance (Jiang et al. [12]; Rapach et al. [26]; Wang et al. [28]). Consequently, we next examine the statistical performance and economic gains of the IQR constraint model and the original model over the business cycle. $R_{\mathrm{OS}}^{2}$ based on recession and expansion periods are given as follows:

$$
R_{\mathrm{OS}, c}^{2}=1-\frac{\sum_{t=M+1}^{T}\left(r_{t}-\widehat{r}_{i, t}\right)^{2} I_{t}^{c}}{\sum_{t=M+1}^{T}\left(r_{t}-\widehat{r}_{i, t}\right)^{2} I_{t}^{c}}, \quad \text { for } c=\mathrm{EXP}, \mathrm{REC},
$$

where $I_{t}^{c}(c=\mathrm{EXP}, \mathrm{REC})$ is an indicator variable equal to one when month $t$ is within the scope of the expansion (recession) period and zero otherwise. The expansion and recession period of the NBER-dated business cycle are derived from the FRED database.

This table shows the out-of-sample statistical performance and economic performance of the original (no constraint) model and quartile difference constraint model based on 14 macroeconomic predictors over business cycles. We evaluate the accuracy of out-of-sample stock return forecast by employing $R_{\mathrm{OS}}^{2} * * *, * *$, and $*$ mean significance at the $1 \%, 5 \%$, and $10 \%$ levels, respectively. We employ the certainty equivalent return (CER) gains to remark the performance of the portfolio. CER is a utilityrelated indicator which is derived from investors investing their assets between stock and T-bill rate. We use risk aversion coefficient equal to 3 and limit the weight to -0.5 to 1.5 to calculate the CER. Furthermore, the CER gains should be multiplied by 1200 as an annualized percentage value.

Table 6 reports $R_{\mathrm{OS}}^{2}$ and CER gains for the original model as well as the IQR constraint model during the recession and expansion period. As can be seen from the second to fifth columns of this table, the forecasting performance in the period of recession is much better than that in the period of expansion, which is consistent with the conclusion of the previous article (Neely et al. [10]; Pan et al. [23]). Furthermore, in either the recession or expansion period, the IQR constraint model can yield larger $R_{\mathrm{OS}}^{2}$ than the original model in every univariate forecasting regression. Particularly, in the period of recession, we can obtain 11 positive $R_{\mathrm{OS}}^{2}$ after implementing the IQR constraint model. For the period of expansion, our constraint strategy leads the number of positive $R_{\mathrm{OS}}^{2}$ to increase from 1 to 9 .

In terms of economic performance, the difference between expansion and recession is similar to that of statistical performance. During the recession, almost all individual predictor forecasting models realize positive CER gains based on the IQR constraint model, and nearly all CER gains of the IQR constraint model are much larger than the original model. When considering economic performance from the perspective of the expansion period, although the economic performance of all the predictors seems to be not as good as the recession, 13 of the 14 CER gains have made progress after the implementation of the IQR constraint model. In a word, the prediction results of the expansion period and recession period show that rational investors will achieve more accurate prediction information and better economic value compared with the original model and historical average benchmark model with the application of the IQR constraint model.

5.2. Alternative Parameters for CER. For a portfolio exercise, the optimal portfolio weight is often affected by the risk aversion coefficient, the weight range allocated to the stock investment, and the transaction cost. Consequently, we employ some alternative parameters to create portfolio performance for stock return prediction. Specifically, we apply $\gamma=2,4$, and 6 instead of $\gamma=3$ to verify the stability of portfolio return. In addition, we consider limiting the weight range to 0 to 1.5 instead of -0.5 to 1.5 as previously used. Furthermore, we followed Neely et al. [10] who consider a proportional transaction cost; that is, the value of each transaction is 50 basis points rather than 0 bases.

This table shows the out-of-sample portfolio performance of the original (no constraint) model and constraint models based on 14 macroeconomic predictors. We employ the certainty equivalent return (CER) gains to remark the performance of the portfolio. CER is a utility-related indicator which is derived from investors investing their assets between stock and T-bill rate. In this table, we use risk aversion coefficient (i.e., $\gamma=2,4,6$ ), the weight range (i.e., $\omega=[0,1.5])$ of substitution, and the alternative transaction cost (i.e., $\mathrm{TC}=50$ ) to calculate the CER. The specific 
TABle 6: Portfolio performance and Statistical performance over the business cycle.

\begin{tabular}{|c|c|c|c|c|c|c|c|c|}
\hline & \multicolumn{4}{|c|}{ Statistical performance } & \multicolumn{4}{|c|}{ Economic performance } \\
\hline & \multicolumn{2}{|c|}{ Recession period } & \multicolumn{2}{|c|}{ Expansion period } & \multicolumn{2}{|c|}{ Recession period } & \multicolumn{2}{|c|}{ Expansion period } \\
\hline & Original & IQR & Original & IQR & Original & IQR & Original & IQR \\
\hline$\overline{\mathrm{DP}}$ & $1.779^{* *}$ & $2.459^{* *}$ & -0.727 & 0.016 & 6.655 & 7.243 & -3.116 & -3.015 \\
\hline DY & $2.696^{* * *}$ & $3.301^{* * *}$ & -1.477 & -0.823 & 12.141 & 12.216 & -4.322 & -4.322 \\
\hline $\mathrm{EP}$ & -2.199 & -1.780 & $-1.203^{*}$ & $-0.445^{* *}$ & 9.467 & 9.467 & -1.100 & -0.951 \\
\hline $\mathrm{DE}$ & -2.641 & -2.013 & -1.041 & -0.229 & -1.090 & -0.152 & -0.128 & 0.079 \\
\hline SVAR & 0.662 & 0.838 & -0.024 & 0.835 & 0.304 & 1.284 & -0.288 & 0.036 \\
\hline $\mathrm{BM}$ & -0.374 & 0.188 & -1.853 & -1.148 & 9.560 & 9.560 & -4.144 & -4.090 \\
\hline NTIS & -3.716 & -2.511 & $0.454^{* *}$ & $1.309^{* *}$ & -0.036 & 0.948 & 0.614 & 0.903 \\
\hline TBL & 1.288 & $2.127^{*}$ & -0.364 & $0.393^{*}$ & 9.782 & 10.774 & 0.113 & 0.249 \\
\hline LTY & 0.587 & 1.341 & -1.123 & $-0.379^{*}$ & 8.379 & 9.367 & -0.672 & -0.554 \\
\hline LTR & 0.877 & $1.462^{*}$ & -1.339 & -0.404 & 7.975 & 8.041 & -0.651 & -0.327 \\
\hline TMS & 0.888 & $1.789^{* *}$ & -0.174 & 0.714 & 8.185 & 9.178 & 0.435 & 0.760 \\
\hline DFY & -0.172 & 0.640 & -0.167 & 0.631 & 1.958 & 2.941 & -0.790 & -0.596 \\
\hline DFR & -0.587 & 0.076 & -0.071 & 0.690 & 1.681 & 2.350 & 0.463 & 0.630 \\
\hline INFL & -0.419 & 0.374 & 0.066 & 0.851 & 2.927 & 3.907 & -0.212 & -0.038 \\
\hline
\end{tabular}

operation is that each time one parameter is changed, the other parameters maintain the original basic parameters (i.e., $\gamma=3, \omega=[-0.5,1.5], \mathrm{TC}=0$ ). Furthermore, the CER gains should be multiplied by 1200 as an annualized percentage value.

In Table 7, we report the main results for portfolio performance linked to the alternative parameters. It is worth noting that we need to keep the remaining parameters consistent with the previously mentioned parameters when one of the parameters changes. From the first column to the sixth column of this table, we can see that although the CER gains of the original model and the IQR constraint model have obvious differences under different risk aversion coefficients, the economic performance of all of the predictors based on the IQR constraint model significantly outperforms the original model. In addition, when the risk aversion coefficient equals 2,4 , and 6 , we can obtain 10,9 , and 9 positive CER gains after the application of the IQR constraint model, respectively, which is consistent with the results of Table 2 .

Table 7 also suggests that the economic performance of all predictors related to our constraint model has improved after limiting the weight range from 0 to 1.5 , and 10 positive CER gains have been generated. Finally, when we consider the transaction cost (i.e., $\mathrm{TC}=50$ ), all CER gains fail to beat the ones compared with nontransaction costs. This is because of the stability of the historical average; however, economic performance is inversely proportional to the historical average. Inevitably, our forecasts increase the frequency of transactions, which in turn increases transaction costs. Nevertheless, we can still improve the CER gains of all predictors with the implementation of the IQR constraint model relative to the original model.

According to the above analyses, the economic gains are robust based on the IQR constraint model after applying the alternative parameters to CER.

5.3. Multivariate Information Analysis. Univariate forecasting model usually has model uncertainty, even if the economic constraint model may also have model uncertainty. To solve this issue, the existing pieces of literature have developed strand multivariate combination approaches to predict stock returns (see, e.g., Neely et al. [10], Dai and Zhu [25], and Rapach et al. [26]). The combination approach that we mainly use is proposed by Rapach et al. [26], which is given as follows:

$$
\widehat{r}_{t+1}^{\mathrm{com}}=\sum_{i=1}^{N} \omega_{i, t} \widehat{r}_{i, t+1},
$$

where $N(N=14)$ denotes the number of predictors; $\widehat{r}_{i, t+1}$ represents the out-of-sample prediction result of OLS estimation of the $i$ th predictor; $\omega_{i, t}$ is the combined weight corresponding to the predictor; and $\widehat{r}_{t+1}^{\mathrm{com}}$ denotes the combination prediction for month $t+1$. Next, following Zhang et al. [51, 52], we adopt five combination methods corresponding to different weight strategy.

This table shows the out-of-sample statistics performance of combination forecast. We evaluate the accuracy of out-of-sample stock return forecast by employing $R_{\mathrm{OS}}^{2} \cdot R_{\mathrm{OS}}^{2}$ computes the reduction in MSPE (mean squared prediction error) for the predictive regression model relative to the benchmark model. The statistical significance of $R_{\mathrm{OS}}^{2}$ is examined by employing Clark and West [24] test. $* * *, * *$, and $*$ mean significance at the $1 \%, 5 \%$, and $10 \%$ levels, respectively. The out-of-sample period of this table is 1948 : $01-2018: 12$.

Table 8 shows that the prediction performance derived from combination forecast approaches is significantly superior to the historical average, which is consistent with antecedent literature related to stock return prediction (see, e.g., Rapach et al. [26]; Wang et al. [28]). In addition to CT constraint that fails to beat the original model and CDA strategy, our IQR constraint model and the corresponding combination model can all surpass the original model. Notably, our IQR constraint model as well as the mixed models related to IQR constraint model can generate $R_{\mathrm{OS}}^{2}$ of more than $1 \%$, while other models or combination models can hardly yield $R_{\mathrm{OS}}^{2}$ exceeding $1 \%$, which once again 
TABLE 7: Alternative parameters about CER.

\begin{tabular}{|c|c|c|c|c|c|c|c|c|c|}
\hline \multicolumn{2}{|c|}{$\gamma=2$} & \multicolumn{2}{|c|}{$\gamma=4$} & \multicolumn{2}{|c|}{$\gamma=6$} & \multicolumn{2}{|c|}{$\omega=[0,1.5]$} & \multicolumn{2}{|c|}{$\mathrm{TC}=50$} \\
\hline Original & IQR & Original & IQR & Original & IQR & Original & IQR & Original & IQR \\
\hline-2.911 & -2.698 & -0.890 & -0.750 & 0.232 & 0.342 & -1.127 & -1.013 & -1.945 & -1.789 \\
\hline-3.535 & -3.519 & -1.159 & -1.151 & -0.051 & -0.031 & -1.141 & -1.130 & -2.379 & -2.370 \\
\hline 0.240 & 0.404 & 0.336 & 0.445 & 0.341 & 0.430 & 0.537 & 0.608 & 0.135 & 0.256 \\
\hline-0.337 & 0.010 & -0.266 & -0.018 & -0.571 & -0.389 & -0.207 & 0.048 & -0.303 & -0.025 \\
\hline-0.077 & 0.299 & -0.332 & 0.118 & -0.478 & -0.020 & -0.202 & 0.159 & -0.282 & 0.108 \\
\hline-2.896 & -2.854 & -1.898 & -1.851 & -1.513 & -1.466 & -1.428 & -1.428 & -2.449 & -2.404 \\
\hline 0.294 & 0.671 & -0.034 & 0.328 & -0.254 & 0.088 & 0.530 & 0.862 & 0.368 & 0.711 \\
\hline 0.751 & 1.008 & 1.253 & 1.515 & 0.974 & 1.190 & 1.479 & 1.680 & 1.330 & 1.555 \\
\hline-0.043 & 0.191 & 0.724 & 0.938 & 0.602 & 0.761 & 0.959 & 1.144 & 0.434 & 0.646 \\
\hline 0.476 & 0.756 & 0.299 & 0.593 & -0.103 & 0.110 & 0.591 & 0.820 & -1.083 & -0.798 \\
\hline 1.443 & 1.820 & 1.184 & 1.591 & 0.554 & 0.903 & 1.555 & 1.917 & 1.299 & 1.670 \\
\hline-0.788 & -0.457 & -0.701 & -0.402 & -0.775 & -0.478 & -0.361 & -0.111 & -0.494 & -0.225 \\
\hline 0.334 & 0.630 & 0.435 & 0.628 & 0.331 & 0.476 & 0.569 & 0.751 & -0.020 & 0.213 \\
\hline-0.083 & 0.222 & 0.180 & 0.466 & 0.076 & 0.289 & 0.272 & 0.504 & -0.466 & -0.213 \\
\hline
\end{tabular}

TABLE 8: Performance of multivariate strategies evaluated by $R_{\mathrm{OS}}^{2}$.

\begin{tabular}{|c|c|c|c|c|c|c|c|}
\hline & Original & $\mathrm{CT}$ & $\mathrm{CDA}$ & IQR & CDA\&CT & CT\&IQR & CDA\&IQR \\
\hline Mean & $0.515^{* * *}$ & $0.424^{* *}$ & $0.609^{* * *}$ & $1.275^{* *}$ & $0.535^{* * *}$ & $1.200^{* *}$ & $1.363^{* *}$ \\
\hline Median & $0.396^{* * *}$ & $0.396^{* * *}$ & $0.397^{* * *}$ & $1.143^{*}$ & $0.397^{* * *}$ & $1.143^{*}$ & $1.152^{*}$ \\
\hline Trimmed mean & $0.474^{* * *}$ & $0.436^{* * *}$ & $0.509^{* * *}$ & $1.235^{* *}$ & $0.503^{* * *}$ & $1.201^{* *}$ & $1.260^{* *}$ \\
\hline DMSPE(0.9) & $0.539^{* * *}$ & $0.440^{* * *}$ & $0.611^{* * *}$ & $1.296^{* *}$ & $0.541^{* * *}$ & $1.213^{* *}$ & $1.362^{* *}$ \\
\hline DMSPE(1) & $0.503^{* * *}$ & $0.413^{* *}$ & $0.608^{* * *}$ & $1.262^{* *}$ & $0.535^{* * *}$ & $1.189^{* *}$ & $1.361^{* *}$ \\
\hline
\end{tabular}

TABLE 9: Out-of-sample forecast results (1982:01-2018:12).

\begin{tabular}{|c|c|c|c|c|c|c|c|}
\hline \multirow[b]{2}{*}{ Predictor } & \multirow[b]{2}{*}{ Original } & \multicolumn{3}{|c|}{ Existing model } & \multicolumn{3}{|c|}{ Mixed model } \\
\hline & & CT & $\mathrm{CDA}$ & IQR & $\mathrm{CDA} \& \mathrm{CT}$ & CT\&IQR & CDA\&IQR \\
\hline $\mathrm{DP}$ & -1.030 & -0.726 & -0.309 & 0.283 & -0.231 & 0.587 & 0.883 \\
\hline DY & -1.552 & -0.828 & -0.435 & -0.394 & -0.396 & 0.422 & 0.835 \\
\hline $\mathrm{EP}$ & -1.195 & 0.109 & -0.416 & 0.015 & 0.096 & $1.384^{*}$ & 0.828 \\
\hline $\mathrm{DE}$ & -0.526 & -0.078 & -0.237 & 0.855 & 0.322 & 1.303 & 1.025 \\
\hline SVAR & 0.345 & 0.204 & 0.273 & $1.577^{*}$ & 0.183 & $1.615^{*}$ & $1.663^{*}$ \\
\hline $\mathrm{BM}$ & -1.895 & -1.023 & -0.390 & -0.688 & -0.343 & 0.207 & 0.844 \\
\hline NTIS & -1.282 & -1.281 & -1.367 & 0.428 & -1.367 & 0.429 & 0.306 \\
\hline TBL & -0.582 & -0.483 & -0.106 & 0.823 & -0.102 & 0.923 & 1.321 \\
\hline LTY & -0.787 & -0.374 & $1.503^{* *}$ & 0.562 & $1.119^{* *}$ & 0.975 & $2.758^{* *}$ \\
\hline LTR & 0.146 & 0.098 & $1.114^{* *}$ & $1.671^{*}$ & $1.095^{* *}$ & $1.623^{*}$ & $2.495^{* *}$ \\
\hline TMS & -0.413 & -0.413 & 0.190 & $1.198^{*}$ & 0.190 & $1.198^{*}$ & $1.675^{*}$ \\
\hline DFY & -0.115 & -0.115 & -0.062 & 1.335 & -0.062 & 1.335 & 1.380 \\
\hline DFR & 0.270 & -0.103 & -0.260 & 1.599 & -0.242 & 1.226 & 1.171 \\
\hline INFL & -0.368 & -0.368 & -0.293 & 1.054 & -0.293 & 1.054 & 1.137 \\
\hline
\end{tabular}

manifest that our IQR constraint model can improve prediction performance.

5.4. Alternative Out-of-Sample Period. In this section, we apply an alternative out-of-sample period to evaluate the forecasting ability of the prediction model. Previous pieces of literature (Rapach et al. [13]; li et al. [53]) have shown that the choice of prediction period will impact the out-ofsample forecasting performance of the forecasting model. As a result, we consider an out-of-sample prediction period from 1982: 01 to $2018: 12$, to further examine the predictive ability of the IQR constraint model.

This table shows the out-of-sample statistical performance of the original (no constraint) model, existing constraint models, and mixed constraint models based on 14 macroeconomic predictors. We evaluate the accuracy of outof-sample stock return forecast by employing $R_{\mathrm{OS}}^{2} \cdot R_{\mathrm{OS}}^{2}$ computes the reduction in MSPE (mean squared prediction error) for the predictive regression model relative to the benchmark model. The statistical significance of $R_{\mathrm{OS}}^{2}$ is examined by employing Clark and West [24] test. $* * *, * *$, 
and * mean significance at the $1 \%, 5 \%$, and $10 \%$ levels, respectively. The out-of-sample period of this table is 1982 : 01-2018:12.

Table 9 reports the statistical performance of the existing models and the mixed models in the alternative out-ofsample period. The second column in this table is the out-ofsample $R_{\mathrm{OS}}^{2}$ of the original model, which is used to compare with the model applied earlier in this paper. We find that the CT constraint model and the CDA strategy can improve the forecasting performance of 7 and 8 predictors, respectively. Notably, with the application of the IQR constraint model, all predictors can generate larger $R_{\mathrm{OS}}^{2}$, and most of them are positive numbers. What is more, based on the mixed model, almost all variables achieve larger $R_{\mathrm{OS}}^{2}$, especially the mixed model that combines the IQR constraint model. More specially, the CT\&IQR model and the CDA\&IQR model lead $R_{\mathrm{OS}}^{2}$ of all variables to become positive, where $R_{\mathrm{OS}}^{2}$ of LTY changes the most ( $R_{\mathrm{OS}}^{2}$ increases from $-0.787 \%$ to $2.758 \%$ ). Overall, there is more accurate prediction information in predicting stock returns by applying the IQR constraint model and its mixed model.

\section{Conclusion}

We construct a new approach based on the principle of boxplot to constrain stock return forecasts. This approach imposes upper and lower bound to achieve reasonable return forecasts. As abnormal prediction information is usually accompanied by high risk, it is difficult for investors to believe abnormal prediction information. Fortunately, our approach not only identifies outliers but also eliminates outliers. In order to better demonstrate the predictability of our new constraint approach, we compare two constraint approaches previously proposed by Campbell and Thompson [17] and Yi et al. [20]. The out-of-sample empirical results exhibit that from the perspective of statistics or economics, our new constraint approach can archive greater economic gains compared with the competition model as well as the unconstraint approach. In addition, we also analyze the predictability and economic gains of constraint and unconstraint approaches from combination model, multivariate strategy, alternative parameters (i.e., $\gamma=2,4,6, \omega=[0,1.5]$, and $\mathrm{TC}=0$ ), business cycles, and alternative out-of-sample period. All empirical results demonstrate that our constraint method is robust and more stable than the unconstraint model and other competition constraint models.

\section{Data Availability}

The data or materials used to support the findings of this study will be available from the corresponding author upon request.

\section{Conflicts of Interest}

The authors declare that they have no conflicts of interest.

\section{Acknowledgments}

The authors would like to express their gratitude for the support given by the National Natural Science Foundation of China (no. 71771030).

\section{References}

[1] J. Y. Campbell and R. J. Shiller, "Stock prices, earnings, and expected dividends," Journal of Finance, vol. 43, no. 3, pp. 661-676, 2008.

[2] J. Pontiff and L. D. Schall, "Book-to-market ratios as predictors of market returns," Journal of Financial Economics, vol. 49, no. 2, pp. 141-160, 1998.

[3] Z. Dai, J. Kang, and F. Wen, "Predicting stock returns: a risk measurement perspective," International Review of Financial Analysis, vol. 74, Article ID 101676, 2021.

[4] Z. Dai, X. Dong, J. Kang, and L. Hong, "Forecasting stock market returns: new technical indicators and two-step economic constraint method," The North American Journal of Economics and Finance, vol. 53, Article ID 101216, 2020.

[5] W. E. Ferson and C. R. Harvey, "The risk and predictability of international equity returns," Review of Financial Studies, vol. 6, no. 3, pp. 527-566, 1993.

[6] I. Welch and A. Goyal, "A comprehensive look at the empirical performance of equity premium prediction," Review of Financial Studies, vol. 21, no. 4, pp. 1455-1508, 2008.

[7] L. Liu, F. Ma, and Y. Wang, "Forecasting excess stock returns with crude oil market data," Energy Economics, vol. 48, pp. 316-324, 2015.

[8] T. Bollerslev, G. Tauchen, and H. Zhou, "Expected stock returns and variance risk premia," Review of Financial Studies, vol. 22, no. 11, pp. 4463-4492, 2009.

[9] T. Bollerslev, J. Marrone, L. Xu, and H. Zhou, "Stock return predictability and variance risk premia: statistical inference and international evidence," Journal of Financial and Quantitative Analysis, vol. 49, no. 3, pp. 633-661, 2014.

[10] C. J. Neely, D. E. Rapach, J. Tu, and G. Zhou, "Forecasting the equity risk premium: the role of technical indicators," Management Science, vol. 60, no. 7, pp. 1772-1791, 2014.

[11] J. Brogaard and A. Detzel, "The asset-pricing implications of government economic policy uncertainty," Management Science, vol. 61, no. 1, pp. 3-18, 2015.

[12] F. Jiang, J. Lee, X. Martin, and G. Zhou, "Manager sentiment and stock returns," Journal of Financial Economics, vol. 132, no. 1, pp. 126-149, 2019.

[13] D. E. Rapach, M. C. Ringgenberg, and G. Zhou, "Short interest and aggregate stock returns," Journal of Financial Economics, vol. 121, no. 1, pp. 46-65, 2016.

[14] A. Manela and A. Moreira, "News implied volatility and disaster concerns," Journal of Financial Economics, vol. 123, no. 1, pp. 137-162, 2017.

[15] M. H. Pesaran and A. Timmermann, "Predictability of stock returns: robustness and economic significance," The Journal of Finance, vol. 50, no. 4, pp. 1201-1228, 1995.

[16] A. Ang and M. Piazzesi, "A no-arbitrage vector autoregression of term structure dynamics with macroeconomic and latent variables," Journal of Monetary Economics, vol. 50, no. 4, pp. 745-787, 2003.

[17] J. Y. Campbell and S. B. Thompson, "Predicting excess stock returns out of sample: can anything beat the historical 
average?" Review of Financial Studies, vol. 21, no. 4, pp. 1509-1531, 2008.

[18] L. Pástor and R. F. Stambaugh, "Predictive systems: Living with imperfect predictors," The Journal of Finance, vol. 64, no. 4, pp. 1583-1628, 2009.

[19] Ľ. Pástor and R. F. Stambaugh, "Are stocks really less volatile in the long run?" The Journal of Finance, vol. 67, no. 2, pp. 431-478, 2012.

[20] Y. Yi, F. Ma, Y. Zhang, and D. Huang, "Forecasting stock returns with cycle-decomposed predictors," International Review of Financial Analysis, vol. 64, pp. 250-261, 2019.

[21] D. Pettenuzzo, A. Timmermann, and R. Valkanov, "Forecasting stock returns under economic constraints," Journal of Financial Economics, vol. 114, no. 3, pp. 517-553, 2014.

[22] D. Rapach and G. Zhou, "Forecasting stock returns," Handbook of Economic Forecasting, Elsevier, Amsterdam, Netherlands.

[23] Z. Dai and X. M. Chang, "Forecasting stock market volatility: can the risk aversion measure exert an important role?" North American Journal of Economics and Finance, 2021.

[24] T. E. Clark and K. D. West, "Approximately normal tests for equal predictive accuracy in nested models," Journal of Econometrics, vol. 138, no. 1, pp. 291-311, 2007.

[25] Z. Dai and H. Zhu, "Stock return predictability from a mixed model perspective," Pacific-Basin Finance Journal, vol. 60, Article ID 101267, 2020.

[26] D. E. Rapach, J. K. Strauss, and G. Zhou, "Out-of-sample equity premium prediction: combination forecasts and links to the real economy," Review of Financial Studies, vol. 23, no. 2, pp. 821-862, 2010.

[27] Z. Dai and J. Kang, "Bond yield and crude oil prices predictability," Energy Economics, vol. 97, Article ID 105205, 2021.

[28] Z. Dai and R. J. Shi, "Forecasting commodity prices with technical indicators: A partial least squares approach," International Review of Economics and Finance, 2021.

[29] Z. Dai, H. Zhuo, J. Kang, and F. Wen, "The skewness of oil price returns and equity premium predictability," Energy Economics, vol. 94, Article ID 105069.

[30] N. Devpura, P. K. Narayan, and S. S. Sharma, "Is stock return predictability time-varying?" Journal of International Financial Markets, Institutions and Money, vol. 52, pp. 152-172, 2018.

[31] A. Goyal and I. Welch, "Predicting the equity premium with dividend ratios," Management Science, vol. 49, no. 5, pp. 639-654, 2003.

[32] R. Hammerschmid and H. Lohre, "Regime shifts and stock return predictability," International Review of Economics \& Finance, vol. 56, pp. 138-160, 2018.

[33] Z. Dai, X. Chen, and F. Wen, “A modified Perry's conjugate gradient method-based derivative-free method for solving large-scale nonlinear monotone equations," Applied Mathematics and Computation, vol. 270, pp. 378-386, 2015.

[34] D. Huang, F. Jiang, J. Tu, and G. Zhou, "Investor sentiment aligned: a powerful predictor of stock returns," Review of Financial Studies, vol. 28, no. 3, pp. 791-837, 2015.

[35] X. Yang, S. Wen, Z. Liu, C. Li, and C. Huang, "Dynamic properties of foreign exchange complex network," Mathematics, vol. 7, no. 9, p. 832, 2019.

[36] Y. Zhang, F. Ma, and Y. Wang, "Forecasting crude oil prices with a large set of predictors: can LASSO select powerful predictors?" Journal of Empirical Finance, vol. 54, pp. 97-117, 2019.
[37] Z. Dai and H. Zhu, "A modified Hestenes-Stiefel-type derivative-free method for large-scale nonlinear monotone equations," Mathematics, vol. 8, no. 2, p. 168, 2020.

[38] D. Buncic and M. Tischhauser, "Macroeconomic factors and equity premium predictability," International Review of Economics \& Finance, vol. 51, pp. 621-644, 2017.

[39] F. X. Diebold and R. S. Mariano, "Comparing predictive accuracy," Journal of Business \& Economic Statistics, vol. 13, no. 3, pp. 253-263, 1995.

[40] Z. Dai and J. Kang, "Some new efficient mean-variance portfolio selection models," International Journal of Finance \& Economics, vol. 2021, pp. 1-13, 2021.

[41] B. Kelly and S. Pruitt, "Market expectations in the crosssection of present values," The Journal of Finance, vol. 68, no. 5, pp. 1721-1756, 2013.

[42] A. Bahrami, A. Shamsuddin, and K. Uylangco, "Are advanced emerging market stock returns predictable? A regimeswitching forecast combination approach," Pacific-Basin Finance Journal, vol. 55, pp. 142-160, 2019.

[43] R. Tibshirani, "Regression shrinkage and selection via the lasso," Journal of the Royal Statistical Society: Series B (Methodological), vol. 58, no. 1, pp. 267-288, 1996.

[44] H. Zou and T. Hastie, "Regularization and variable selection via the elastic net," Journal of the Royal Statistical Society: Series B (Statistical Methodology), vol. 67, no. 2, pp. 301-320, 2005.

[45] F. Min, F. Wen, J. Xu, and N. Wu, "Credit supply, house prices, and financial stability," International Journal of Finance \& Economics, pp. 1-21, 2021.

[46] L. Zhao, F. Wen, and X. Wang, "Interaction among China carbon emission trading markets: nonlinear Granger causality and time-varying effect," Energy Economics, vol. 91, Article ID 104901, 2020.

[47] F. Wen, N. Wu, and X. Gong, "China's carbon emissions trading and stock returns," Energy Economics, vol. 86, Article ID 104627, 2020.

[48] Y. Zhang, F. Ma, and Y. Liao, "Forecasting global equity market volatilities," International Journal of Forecasting, vol. 36, no. 4, pp. 1454-1475, 2020.

[49] J. H. Cochrane, "The dog that did not bark: a defense of return predictability," Review of Financial Studies, vol. 21, no. 4, pp. 1533-1575, 2008.

[50] E. F. Fama and K. R. French, "Business conditions and expected returns on stocks and bonds," Journal of Financial Economics, vol. 25, no. 1, pp. 23-49, 1989.

[51] Y. Zhang, F. Ma, B. Shi, and D. Huang, "Forecasting the prices of crude oil: an iterated combination approach," Energy Economics, vol. 70, pp. 472-483, 2018.

[52] Y. Zhang, Y. Wei, Y. Zhang, and D. Jin, "Forecasting oil price volatility: forecast combination versus shrinkage method," Energy Economics, vol. 80, pp. 423-433, 2019.

[53] J. Li and I. Tsiakas, "Equity premium prediction: the role of economic and statistical constraints," Journal of Financial Markets, vol. 36, pp. 56-75, 2017. 